\title{
Prevalencia de deficiencia de hierro y yodo, y parasitosis en niños de Arandas, J alisco, México
}

Edgar Manuel Vásquez-Garibay, $\mathrm{Dr}$ en $\mathrm{C}_{,^{(1)}}$ Enrique Romero-Velarde, $\mathrm{M}$ en $\mathrm{C}_{\left({ }^{(1)}\right.}$ Francisco N ápoles-Rodríguez, MC SP, (1) María Eugenia N uño-C osío, Lic TS, ${ }^{(3)}$ Francisco Trujillo-C ontreras, Dr en C, ${ }^{(2)} 0$ scar Sánchez-Mercado, MC. ${ }^{(4)}$

\section{Vásquez-Garibay EM, Romero-Velarde E, Nápoles-Rodríguez F, Nuño-Cosío ME, Trujillo-Contreras F, Sánchez-Mercado 0. Prevalencia de deficiencia de hierro y yodo, y parasitosis en niños de Arandas, Jalisco, México. Salud Publica Mex 2002;44:195-200.}

El texto completo en inglés de este artículo está disponible en: http://www.insp.mx/salud/index.html

\section{Resumen}

Objetivo. Estimar la prevalencia de deficiencia de hierro, yodo y parasitosis en niños que asisten al Instituto Alteño para el Desarrollo de Jalisco (Inadej), A randas, Jalisco, México. Material y métodos. Estudio transversal efectuado entre 1997 y 1999 con 432 niños de 12 a 120 meses de edad, de nuevo ingreso al Inadej. Se determinaron variables hematoló gicas, yodo en orina y presencia de parásitos. Se utilizaron las pruebas Ji cuadrada y t de Student en variables no paramétricas y paramétricas. Resultados Hubo más anemia (20 vs 7.4\% $p=0.007$ ) y deficiencia de hierro (60.9 vs $44.4 \% p=0.02$ ) en prescolares que en escolares. El $29 \%$ presentaron deficiencia de yodo ( $10.5 \%$ moderada 0 grave) y $47.2 \%$ parasitosis. Predominaron G. lamblia y E. histolytica. Bajo salario, sexo masculino y no tener seguridad social se aso ciaron con parasitosis. Conclusiones. La elevada prevalencia de deficiencia de hierro, yodo y parasito sis obliga al sector salud estatal a ejecutar medidas eficaces para abatir estas enfermedades prevenibles. El texto completo en in-

\author{
Vásquez-Garibay EM, Romero-Velarde E, \\ Nápoles-Rodríguez F, Nuño-Cosío ME, \\ Trujillo-Contreras F, Sánchez-Mercado 0. \\ Prevalence of iron and iodine deficiency \\ and parasitosis in children \\ from Arandas, Jalisco, Mexico.. \\ Salud Publica Mex 2002;44:195-200. \\ The English version of this paper \\ is available at: http://www.insp.mx/salud/index.html
}

\begin{abstract}
A bstract
Objective To estimate the prevalence of iron deficiency, iodine deficiency and parasitosis in children attending the Instituto Alteño para el Desarrollo de Jalisco ((Highlands Institute for D evelopment of Jalisco State, IN ADEJ),A randas, Jalisco, Mexico. Material and Methods. A cross-sectional study was conducted between 1997 and 1999, among 432 children aged 12 to 120 months attending the IN ADEJ. Measurements included hematological values, urine iodine concentration, and presence of parasites. Student's t test chi square tests were used for parametric and nonparametric analysis. Results. The prevalence figures of anemia ( 20 vs $7.4 \%, p=0.007$ ) and iron deficiency ( 60.9 vs $44.4 \%, p=0.02$ ) were higher in preschool than in school children. lodine deficiency was found in $29 \%(10.5 \%$ moderate or severe) and parasitosis in $47.2 \%$ of children, mainly E. histolytica $(30.2 \%)$ and G. lamblia $(28.9 \%)$. Low income, male gender and lack of social security policy holding were associated to parasitosis. Conclusions. The high
\end{abstract}

(1) Instituto de Nutrición Humana, Guadalajara, Jalisco, México.

(2) Laboratorio de Parasitología, Centro U niversitario de Ciencias de la Salud, U niversidad de Guadalajara, G uadalajara, Jalisco, México.

(3) Instituto Alteño para el D esarrollo de Jalisco,A.C., Guadalajara, Jalisco, México.

(4) Instituto Mexicano del Seguro Social, Guadalajara, Jalisco, México.

Fecha de recibido: 8 de enero de 2001 • Fecha de aceptado: 11 de octubre de 2001

Solicitud de sobretiros: M en C. Edgar M.Vásquez-Garibay, Instituto de N utrición Humana, Unidad de Investigación en Ciencias de la Salud, Edificio Anexo al Hospital Civil “D r. Juan I. Menchaca”, 30. Piso, Salvador Q uevedo y Zubieta \# 750, 44340, S.L. G uadalajara, Jalisco, México. Correo electrónico: inhu@ cucs.udg.mx 
glés de este artículo está disponible en:http://www.insp.mx/ salud/index.html

Palabras clave: niños; deficiencia de hierro; deficiencia de yodo; parasitosis; México prevalence rates of iron deficiency, iodine deficiency, and parasitosis, should be addressed by state health services with effective interventions to restrain these preventable diseases. The English version of this paper is available at: http://www.insp.mx/salud/index.html

Key words: children iron deficiency; lo dine deficiency; parasitosis; México a valoración del estado de nutrición del niño en el L ámbito comunitario, ya se trate de grupos o localidades, normalmente incluye la identificación de indicadores indirectos que dan información sobre las condiciones sociodemográficas, económicas, educacionales y de hábitos de alimentación de la población, así como la obtención de indicadores directos, como las mediciones antropométricas. Sin embargo, por dificultades de diversa índole, es menos frecuente realizar un escrutinio de deficiencias nutricias específicas o aun de parasitosis, entidades clínicas que inciden directamente sobre el estado de nutrición del niño.

Por su magnitud son de particular importancia la deficiencia de hierro, considerada como el problema de origen nutricio de mayor prevalencia en México, ${ }^{1}$ y la deficiencia dietética crónica de yodo (DDCY) de la cual existe muy poca información en el país, ${ }^{2-4}$ y cuya distribución geográfica se ubicaría principalmente en las regiones más áridas y montañosas. El estudio de Noguera $(1994)^{3}$ hace referencia a otro hecho en el estado de Hidalgo y demuestra una prevalencia de $6 \%$ de bocio clínico y, de acuerdo con la excreción urinaria de yodo, 32.8\% de la población estudiada se encuentra en riesgo moderado o grave de deficiencia de yodo. La mayor inquietud que estas dos patologías, con alta prevalencia en México, producen, derivan de que cada una en forma aislada, combinadas o asociadas con desnutrición crónica afectan el crecimiento físico, el desarrollo psicomotor y el rendimiento escolar del niño dejando secuelas probablemente irreversibles. ${ }^{5}$

En el estado de Jalisco hay muy poca información al respecto. Especialmente sobre la DDCY. Según la encuesta para definir áreas con deficiencia de yodo (Endeyo $)^{4}$ la prevalencia de bocio clínico en Jalisco ocupó el $12^{\circ}$ lugar de 13 entidades federativas seleccionadas, con una prevalencia de $1 \%$; y los casos con excreción de yodo anormalmente baja en orina tuvieron una prevalencia de $0.4 \%$. Se incluyeron seis municipios, dos de la región de los Altos, Tepatitlán de Morelos, donde fue detectado un solo caso, y Arandas. Particularmente la región de los Altos de Jalisco, debido a su altitud y a la aridez del suelo, es probable que sea una zona deficiente en yodo. Es conocido que la población que ahí radica consume frecuentemente sal de grano cuya yodación, aunque obligatoria, no ha sido bien documentada.

Por otro lado, se sabe que Jalisco es un estado en donde se observa una prevalencia elevada de parasitosis, particularmente ascariasis y giardiasis. Ambas parasitosis en forma individual pueden provocar una mala absorción intestinal, intolerancia a la lactosa, alteración del apetito y pérdida excesiva de nutrimentos en la materia fecal. Tal condición clínica puede impactar negativamente en mayor o menor grado el estado nutricio del niño. ${ }^{6,7}$

Por lo tanto, el propósito de la presente comunicación es informar sobre la prevalencia de deficiencia de yodo, hierro y parasitosis en la población pediátrica que asiste al Inadej en Arandas, y analizar algunas características generales comunes a los niños que sufren estas patologías.

El Inadej es una institución con carácter de asociación civil no lucrativa, que participa en la solución de los problemas que aquejan a la región de los Altos de Jalisco y tiene una relación estrecha con el gobierno del municipio apoyando sus programas de asistencia. Tiene registrados 1196 niños, 603 de la cabecera municipal de Arandas, y 593 del área rural. Los niños que acuden a este organismo podrían considerarse representativos de la población de escasos recursos del municipio mencionado.

\section{Material y métodos}

Estudio de observación transversal, descriptivo. Se incluyó la población pediátrica que asistía al Inadej del 8 de diciembre de 1997 al 31 de diciembre de 1999. Se incluyeron prescolares y escolares de 12 a 120 meses de edad que asistieron regularmente, que tuvieron peso normal al nacer, sin infección aparente en el último mes, diarrea u otra patología agregada; sin evidencia de enfermedades crónicas (genopatías, malformaciones y cardiopatías congénitas, neumopatías crónicas, síndromes de mala absorción, tuberculosis, etcétera). 
El estudio sociodemográfico, económico y de hábitos de alimentación se efectuó en 432 niños de reciente ingreso, de una población total registrada en el Inadej de 1196 sujetos. Sin embargo, las subpoblaciones de niños para los estudios de yodo, hierro y parasitosis se obtuvieron de manera independiente, de manera aleatorizada del total de la población que había ingresado recientemente $(n=432)$ mediante la tabla de dígitos de Snedecor y Cochran. ${ }^{8}$ De la población total de niños de reciente ingreso se calculó cada una de las submuestras de la siguiente manera: deficiencia de hierro, prevalencia $23 \%,{ }_{;}^{5}$ error alfa $5 \%$ y nivel de confianza $99 \%(n=57)$. Finalmente, se incluyeron 50 sujetos de 12 a 71 meses, y 81 de 72 a 120 meses. Deficiencia crónica de yodo, prevalencia $10 \%,{ }^{5}$ error alfa $5 \%$ y nivel de confianza 99\%, $(n=199)$, parasitosis, prevalencia $19.5 \%$ de ascariasis y giardiasis, ${ }^{9}$ error alfa $5 \%$ y nivel de confianza 99\%, $(n=83)$. Cuando la primera muestra de coproparasitoscópico fue negativa se obtuvo una muestra adicional.

Variables dependientes: 1) Hemoglobina (g/dl); 2) Volumen corpuscular medio (fl); 3) Concentración media de hemoglobina corpuscular ( $\mathrm{g} / \mathrm{dl}) ; 4$ Hemoglobina corpuscular media (pg); 5) Amplitud de distribución de eritrocitos $(\%)$; 6) Ferritina sérica $(\mu \mathrm{g} / 1)$;7) Concentración de yodo en orina; 9) Presencia de parasitosis.

Variables Independientes: 1) Ficha de identificación; 2) Características familiares; 3) Datos económicos; 4) Alimentación familiar; 5) Características de la vivienda.

Dos investigadores y dos técnicos participaron directamente en el trabajo de campo. Con el consentimiento por escrito del familiar se obtuvo la información mediante una entrevista individual, con un instrumento diseñado específicamente para el proyecto. Los estudios médico sociales y la obtención de muestras se hicieron de lunes a viernes.

Laboratorio. A las 08:00 horas, y antes del primer alimento se obtuvieron $5 \mathrm{ml}$ de sangre por venopunción antecubital con microtainer, colocando $2 \mathrm{ml}$ en un tubo con Ethylen Diamine Tetra Acetic Acid (EDTA) como anticoagulante para la biometría hemática, y $3 \mathrm{ml}$ en un tubo seco, para ferritina. La muestra fue guardada en refrigerador a temperatura entre $2^{\circ}$ y $8^{\circ} \mathrm{C}$ hasta su transporte al Laboratorio Clínico del Hospital Civil “Dr. Juan I. Menchaca". La biometría hemática se procesó en el equipo H1 Technicon, con técnica de citometría de flujo. La determinación de ferritina por quimioluminiscencia se hizo en un equipo Access. Se utilizó el siguiente límite de normalidad: anemia, hemoglobina < a $12 \mathrm{~g} / \mathrm{dl}$; deficiencia de ferritina $<10 \mathrm{ng} / \mathrm{ml}$; depleción de ferritina $10-20 \mathrm{ng} / \mathrm{ml} .^{10}$
Coproparasitoscópico. Con técnica de flotación de Faust. Las muestras se guardaron máximo siete días en refrigeración a $2-4{ }^{\circ} \mathrm{C}$. En los casos de evacuaciones aguadas se utilizó una solución preservadora de los huevecillos.

Yodo en orina. Se colectaron en un tubo $5 \mathrm{ml}$ de orina de cada sujeto. Las muestras no requirieron de refrigeración o de la adición de conservadores durante su transporte del municipio de Arandas a la ciudad de Guadalajara. Después se mantuvieron a una temperatura de $6{ }^{\circ} \mathrm{C}$ hasta su análisis el cual se llevó a cabo en el Instituto Nacional de Referencia Epidemiológica de la Secretaría de Salud. Se utilizó la técnica de digestión con ácido clorhídrico y medición por acción catalítica. Los resultados fueron expresados en $\mu \mathrm{g} / 1$ de yodo en orina. Este método detecta niveles bajos de yodo en orina con un coeficiente de variación menor a $10 \%$. Criterios epidemiológicos de deficiencia $\mu \mathrm{g} / 1(<20$ grave; 20-49 moderado; 50-99 leve; $>99$ normal). ${ }^{2}$

La información fue capturada en una base de datos especialmente diseñada para el estudio. El análisis de los datos se realizó con los programas Dbase IV y SSPS.PC. Se calcularon estadísticas descriptivas, porcentajes, promedio, desviaciones estándar. Se obtuvo la prevalencia de deficiencia de yodo, hierro y parasitosis. Se utilizó la prueba de Ji cuadrada en asociaciones no paramétricas y la prueba $t$ de Student para dos grupos independientes. Se rechazó la hipótesis nula con una $p \leq 0.05$

Consideraciones éticas. El estudio no representó un daño para los sujetos y sus familias. Se obtuvo el consentimiento informado de los familiares para las determinaciones en muestras de sangre, orina y materia fecal, explicándose al familiar la finalidad de cada evaluación. Se dieron recomendaciones dietéticas y tratamiento, según el caso, a quien presentó deficiencia de hierro, yodo o parasitosis. Además, se obtuvo la aprobación del Comité de Bioética e Investigación de la Universidad de Guadalajara.

\section{Resultados}

Las características generales de los sujetos, incluyendo las variables sociodemográficas, económicas, de hábitos de alimentación y antropométricas, serán analizadas en otro documento. El cuadro I muestra las variables hematológicas. El promedio de hemoglobina y del volumen corpuscular medio (VCM) fue significativamente menor en prescolares $(p<0.0005)$, mientras que la proporción de prescolares con hemoglobina $<$ de 
$12 \mathrm{~g} / \mathrm{dl}$ y VCM $<75$ fue mayor $(p=0.007$ y $p<0.02$, respectivamente). Además, aceptando como normales niveles de ferritina > de $20 \mathrm{ng} / \mathrm{ml}, 44.4 \%$ de los escolares y $60.9 \%$ de los prescolares se encontraron en un estado de depleción y deficiencia de hierro $(p=0.02)$. Considerando sólo los casos con ferritina $<10 \mathrm{ng} / \mathrm{ml}$ $34.4 \%$ de los sujetos estudiados tuvieron una franca deficiencia de hierro.

La figura 1 muestra el estado nutricio de yodo. Los niveles de yodo en orina fueron anormalmente bajos en $29 \%$ de la población estudiada y de éstos, $10.5 \%$ podrían considerarse con un déficit moderado o grave. Debe señalarse que del grupo total 334 niños (77.5\%) consumieron sal de grano y sólo 97 (22.5\%) sal refinada. Desafortunadamente no tenemos el dato de quienes consumieron sal de grano o refinada.

La prevalencia de parasitosis fue de $47.2 \%$, predominando la E. histolytica (30.2\%) y la G. lamblia $(28.9 \%)$, cuadro II. El ingreso económico menor a un salario mínimo, no contar con seguridad social y la pertenencia al sexo masculino estuvo asociado con parasitosis $(p<0.05)$. Otras variables consideradas importantes, como el piso de la vivienda, la presencia de agua intradomiciliaria, drenaje y la edad del niño no estuvieron asociadas con parasitosis.

\section{Cuadro I}

Prevalencia de anemia y Deficiencia de hierro EN NIÑOS QUE ASISTEN A INADEJ, Arandas, Jalisco, MéXICO, 1997-1999

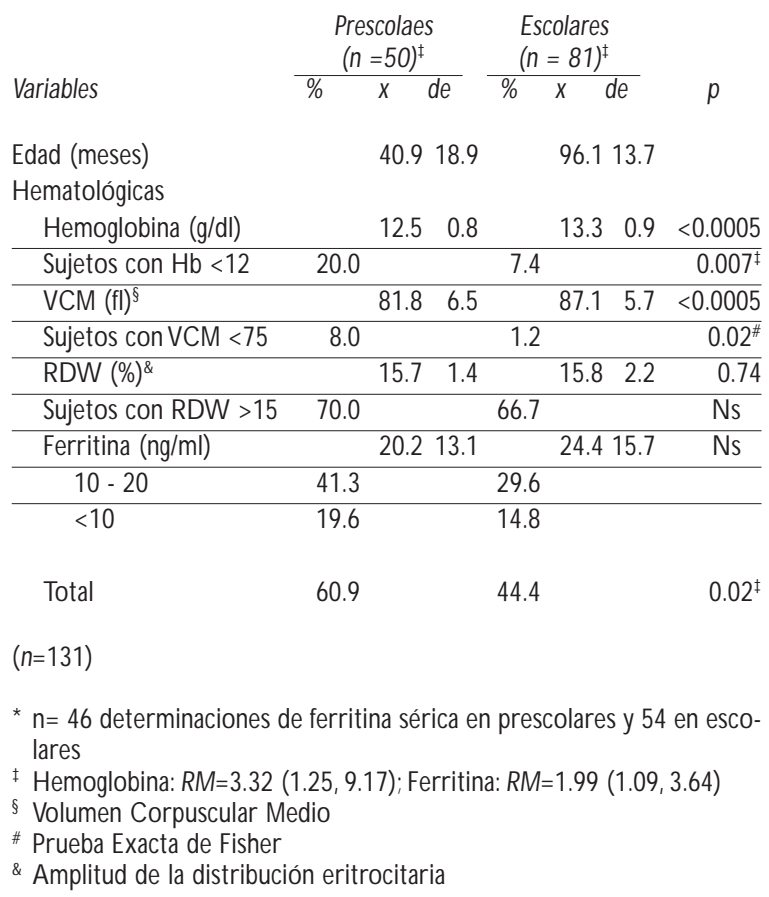

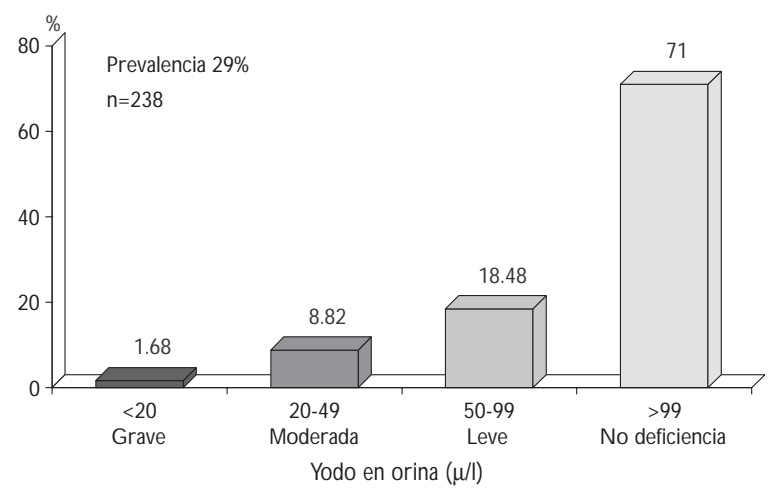

Fuente: Instituto $\mathrm{N}$ acional de Referencia Epidemiológica, SSA (1999) ACC/SCN , 2000

Figura 1. Prevalencia de deficiencia de yodo en niños QUe asisten al Inadej,Arandas, Jalisco, México, 19971999

\section{Cuadro II}

Prevalencia de deficiencia de yodo y parasitosis, Arandas, Jalisco, México, 1997-1999

Variables $n$

\begin{tabular}{lrc}
$\begin{array}{l}\text { Yodo en orina }(\mathrm{mg} / \mathrm{l}) \\
\text { N ormal }\end{array}$ & 169 & 71 \\
\hline D éficit & 69 & 29 \\
\hline Leve $(50-99)$ & 44 & 18.5 \\
\hline Moderada $(20-49)$ & 21 & 8.8 \\
\hline Grave $(<20)$ & 4 & 1.7 \\
\hline Total & 238 & 100
\end{tabular}

Parasitosis*

\begin{tabular}{lcc} 
Positivo & 42 & 47.2 \\
\hline N egativo & 47 & 52.8 \\
\hline Total & 99 & 100
\end{tabular}

Tipo de parásito

E. histolytica $\quad 30.2$

\begin{tabular}{lc} 
E. histolytica & 30.2 \\
\hline G. lamblia & 28.9 \\
\hline E. nana & 26.4 \\
\hline O tros & 14.5 \\
\hline Total & 100
\end{tabular}

* Uno o dos coproparasitoscópicos por sujeto

₹ En algunos sujetos más de un parásito

$\S$ E. Coli, H. nana

\section{Discusión}

La deficiencia de hierro y anemia son los trastornos nutricios más comunes y afectan a más de 3500 millones de personas en el mundo en desarrollo. ${ }^{11}$ Además, es 
un problema grave de salud pública porque debilita el sistema inmunológico y disminuye la capacidad física y mental de la población que la padece. Incluso se ha considerado que, en lactantes y niños pequeños, aun la anemia leve puede dañar el desarrollo intelectual. ${ }^{12-16}$

La prevalencia de deficiencia de hierro observada en el presente estudio en el municipio de Arandas coincide con los datos de UNICEF, ${ }^{17}$ en donde se señala que entre 40 y $50 \%$ de los niños menores de cinco años en el mundo en desarrollo padecen deficiencia de hierro. Los hallazgos del presente estudio también coinciden con aquellos encontrados en la Encuesta Nacional de Nutrición, ${ }^{1}$ en donde se observa una prevalencia elevada de anemia por deficiencia de hierro en prescolares $<$ de 71 meses de edad $(27.2 \%)$, y particularmente elevada durante el segundo año de vida $(50 \%)$. Por ejemplo, debe recordarse que en este estudio, tanto la anemia como la deficiencia de hierro fueron significativamente mayores en prescolares que en escolares. Conviene señalar que entre los hábitos de alimentación observados en esta población pediátrica de Arandas, la ingestión de productos cárnicos, alimentos fundamentales como fuente de hierro heme, es bastante raquítica $(7.9 \%$ consume carne mínimo cinco días a la semana), mientras que el consumo de alimentos como infusiones de té o de café y alimentos ricos en fitatos es más frecuente $(100 \%$ consumen tortillas y $80 \%$ frijoles siete días a la semana) considerándose estos alimentos como factores inhibidores de la absorción de hierro no-heme.

Las enfermedades inducidas por una DDCY constituyen un problema nutricio de magnitud universal. Desde hace décadas se ha reconocido el impacto de la deficiencia de yodo en el desarrollo mental. ${ }^{18}$ En un esfuerzo conjunto de la OMS, UNICEF e International Council for the Control of IODINE Deficiency Disorders (ICCIDD) (por sus siglas en inglés) se presentó recientemente el estado de la DDCY en los ámbitos regional y global. ${ }^{19}$ De los 191 países evaluados, 130 estaban afectados. Cabe mencionar que UNICEF señaló que no tenía datos de DDCY en México. ${ }^{17}$ En los 61 países restantes la DDCY ha sido eliminada. Los datos son insuficientes en 41 países y la mayoría de los países con DDCY proceden del continente africano.

La UNICEF ${ }^{17}$ reconoce que las enfermedades por carencia de yodo son las principales causas de retraso mental en el mundo. De ahí que el hallazgo de la prevalencia de 29\% de DDCY con el criterio de Administrative Committee on Cordination/Sub-Committee on Nutrition Cunited Nations (ACC/SNC) ${ }^{2}$ en la población pediátrica que acude al Inadej en el municipio de Arandas, aunque se trate de un estudio puntual, podría considerarse como un foco rojo de alerta para esta región de los Altos de Jalisco. Además, difiere marcadamente de la prevalencia referida en la Endeyo en los municipios estudiados en Jalisco incluyendo dos de la región de los Altos, Tepatitlán de Morelos y Arandas en donde se encuentra el Inadej. Desde luego que la población estudiada en el presente trabajo no es estadísticamente representativa de esta región, reconocida por la aridez de su tierra y su altitud, pero sí tiene características geográficas, étnicas y sociodemográficas similares. Por ello, es necesario enfatizar la importancia de este hallazgo en materia de salud pública.

Sin embargo, la prevalencia elevada de DDCY en una región del país cuya sal para consumo humano teóricamente está adicionada con yodo y los escasos datos epidemiológicos que existen sobre DDCY, obliga al sector salud a hacer un escrutinio nacional sobre el contenido de yodo en las diferentes presentaciones de sal que se consumen en México, tanto de sal de grano como refinada. Asimismo, es necesario elaborar un diagnóstico de la situación de esta deficiencia nutricia en el ámbito nacional, dadas las consecuencias negativas que tiene la deficiencia de yodo en el desarrollo mental de los seres humanos, cuyo efecto a largo plazo podría tener consecuencias irreversibles.

Como se observó en los resultados, alrededor de $47 \%$ de la población estudiada presentó parasitosis, predominando significativamente la giardiasis y la amibiasis. Ambos tipos de parasitosis, por diferentes mecanismos patogénicos, pueden afectar el estado de nutrición del huésped. La giardiasis es conocida por su capacidad para producir un síndrome de mala absorción intestinal de nutrientes indispensables para el crecimiento y desarrollo del niño, especialmente grasa, lactosa y nutrimentos inorgánicos. ${ }^{6,7}$ Mientras que la E. histolytica, puede transformarse a su forma invasora provocando periodos intermitentes y agudos de diarrea con moco y sangre, ataque al estado general, pérdida de nutrientes y a largo plazo una anemia por pérdidas sanguíneas periódicas. ${ }^{7}$

Ambas parasitosis pueden pasar desapercibidas, o presentarse como procesos subclínicos, afectando el estado general y de nutrición del niño, lo cual aumenta el deterioro de su crecimiento físico y de su desarrollo psicomotor, ya "minado" por la desnutrición crónica y por las deficiencias nutricias específicas como los de hierro y yodo, que en buena medida han resultado de los errores en los hábitos de alimentación.

Desde el punto de vista sociodemográfico y económico hay algunos hallazgos que conviene discutir. Por ejemplo, parece haber mayor riesgo de parasitosis en niños de familias con ingresos económicos inferiores a un salario mínimo mensual, o bien de 
aquellos que no cuentan con seguridad social. Este factor de pertenencia a la seguridad social tendría un efecto protector posiblemente relacionado con la prescripción periódica de antiparasitarios por el médico familiar, situación poco probable en niños de escasos recursos que carecen de esta prestación médica. Asimismo, fue interesante observar que aparentemente los niños tienen mayor riesgo de parasitosis que las niñas. Podría especularse que, a diferencia de las niñas, los niños tienen la costumbre de pasar mayor tiempo en la calle y en consecuencia se exponen con mayor frecuencia al consumo de alimentos de dudosa procedencia, preparados fuera del hogar, sin higiene adecuada y potencialmente contaminados.

En síntesis, la situación sanitaria y nutricia del niño que acude al Inadej, en Arandas, podría considerarse como una verdadera emergencia, no sólo por la elevada prevalencia de desnutrición aguda y crónica, sino por la superposición de parasitosis y deficiencias nutricias específicas (hierro y yodo) que vienen a ensombrecer aún más el pronóstico a largo plazo.

Por lo tanto, es clara la responsabilidad de las autoridades municipales, estatales y, particularmente, de las Secretarías de Salud y Educación Pública para llevar a cabo medidas concretas e inmediatas que reviertan este proceso mórbido que coloca en gran desventaja a los niños afectados por enfermedades potencialmente prevenibles.

\section{Agradecimientos}

Deseamos expresar nuestro agradecimiento a la enfermera Lucía Aguas Avalos por su apoyo en la obtención y conservación de las muestras para el laboratorio; al químico José Guadalupe Alvizo Mora por su apoyo en el procesamiento de los ensayos de laboratorio; a la señorita Zaira Olguín Maciel por su invaluable labor en la preparación de este manuscrito, y a la señora Irma González de González, presidenta del Instituto Alteño para el Desarrollo de Jalisco, por facilitar y apoyar incondicionalmente la realización del proyecto.

\section{Referencias}

1. Instituto N acional de Salud Pública. Encuesta N acional de N utrición 1999. Tomo I. N iños menores de cinco años. Cuernavaca, Morelos: IN SP, 2000.

2. ACC/SNC. Iodine deficiency disorders update. Fourth Report on the W orld N utrition Situation. Ginebra: ACC/SCN in collaboration with International Food Policy Research Institute (IFPRI), 2000: 23-29.

3. N oguera ZA. Eliminar la deficiencia de yodo: un reto de fin de siglo. Bol 0 ficina Sanit Panam 1994; 117(6): 483-494.

4. Dirección General de Epidemiología. Encuesta para definir áreas con deficiencias de yodo. Endeyo. México, D.F.: Secretaría de Salud, 1994.

5. Rosado JL, Bourges H, Saint-Martin B.Vitamin and mineral deficiency in Mexico.A critical review of the state of the art I. Mineral deficiency. Salud Publica Mex 1995; 37(2):130-109.

6. Solomons N W. La lactosa y sus implicaciones en Gastroenterología. Cuadernos de Nutrición 1997;20(4):21-28.

7. Klish W J, Kessler BH, Katz M. Parasitic diseases: Entamoeba histolytica, Giardia lamblia,A scaris lumbricoides. En: 0 ski FA, D eAngelis CC, Feigin RD, W arshaw JB, ed. Principles and Practice of Pediatrics. Philadelphia, (PA): JB Lippincott Com. 1990: 1268-1289.

8. Lilienfeld AM, Lilienfeld DE. Epidemio logía experimental: I. Ensayos clínicos. En: Lilienfeld AM, Lilienfeld DE, ed. Fundamentos de Epidemiología. W ilimington (D elaware):Addison-W esley Iberoamericana, 1987:230-247. 9. Kumate J, Gutiérrez G, Muñoz 0, Santos Jl. Manual de infectología. 3a. edición. México, D.F:: Méndez Editores, 1992:116-127.

10. Fomon SJ. N utrition of normal infants. St. Louis: Missouri Mosby, 1993: 239-260.

11.U nited $\mathrm{N}$ ation's C hildren Fund/U nited $\mathrm{N}$ ations U niversity/W orld $\mathrm{H}$ ealth 0 rganization/Micronutrient Iniciative. Preventing iron deficiency in women and children:Technical consensus on key issues.Technical workshop, october 7-9, Boston and 0 ttawa: International N utrition Foundation and MI, 1999. 12. De Andraca I, Castillo M, W alter T. Psychomotor development and behavior in iron-deficient anemic infants. N utr Rev 1997; 55: 125-132.

13. Pollitt E. Iron deficiency and educational deficiency. Nutr Rev 1997; 55:133-141.

14. Pollitt E. Early iron deficiency and later mental retardation. Am J Clin Nutr 1999; 69: 4-5

15. Lozoff B. Explanatory mechanisms for poorer development in irondeficient anemic infants. En: Pan A merican H ealth 0 rganization, TheW orld Bank, and Tropical Metabolism Research Unit, ed. N utrition, health and child development. 1998 C d.: casa editora, Scientific Publication N 0. 566: 162-178.

16. Hurtado EK, Claussen AH, Scott KG. Early childhood anemia mild or moderate mental retardation. Am J Clin N utr 1999; 69:115-119.

17. Bellamy C. El estado mundial de la infancia 1998. Fondo de las $\mathrm{Na}$ ciones U nidad para la Infancia. N ueva York (N Y): UN IC EF, 1998.

18. Hetzel BS. lodine deficiency disorders (IDD) and their eradication. Lancet 1983; 2: 1126-1129.

19.W HO /UN ICEF/ICCIDD. Progress towards the elimination of iodine deficiency disorders (IDD). Ginebra:W HO, 1999;W HO /N HD/99.4. 